A N N A L E S Annales de Bretagne et des Pays de l'Ouest

Anjou. Maine. Poitou-Charente. Touraine

$119-3 \mid 2012$

Les abbayes martiniennes

\title{
Les prieurés bretons de Marmoutier (XIe-XIIe siècle)
}

The Priories of Marmoutier in Brittany (11th-12th centuries)

Daniel Pichot

\section{(2) OpenEdition}

Journals

Édition électronique

URL : http://journals.openedition.org/abpo/2483

DOI : $10.4000 / a b p o .2483$

ISBN : 978-2-7535-2136-0

ISSN : 2108-6443

Éditeur

Presses universitaires de Rennes

Édition imprimée

Date de publication : 30 octobre 2012

Pagination : 153-175

ISBN : 978-2-7535-2134-6

ISSN : 0399-0826

\section{Référence électronique}

Daniel Pichot, «Les prieurés bretons de Marmoutier (XIe-XIle siècle)», Annales de Bretagne et des Pays de l'Ouest [En ligne], 119-3 | 2012, mis en ligne le 30 octobre 2014, consulté le 01 mai 2019. URL :

http://journals.openedition.org/abpo/2483; DOI : 10.4000/abpo.2483 


\title{
Les prieurés bretons de Marmoutier (XI ${ }^{\mathrm{e}}$-XII ${ }^{\mathrm{e}}$ siècle)
}

\author{
Daniel PICHOT \\ Professeur émérite d'histoire médiévale \\ CERHIO UMR 6258, université Rennes 2
}

L'ampleur du réseau prieural de l'abbaye de Marmoutier a fortement contribué à son rayonnement. Par centaines, l'abbaye de Saint-Martin a installé ses dépendances sur une vaste partie du territoire du royaume de France, plus spécialement dans un grand quart nord-ouest ${ }^{1}$. Les sources, particulièrement abondantes, se dispersent malheureusement dans les dépôts départementaux en raison d'une gestion archivistique assez désastreuse au siècle dernier, ce qui rend les études peu commodes à réaliser². Des cartulaires régionaux ont vu le jour et rendent de grands services mais aucune vue d'ensemble n'a été tentée depuis le travail pionnier, mais aujourd'hui insuffisant, d'O. Gantier ${ }^{3}$. L'ampleur de la tache fait reculer les plus courageux.

Des travaux conduits dans des cadres géographiques restreints dévoilent cependant la politique d'implantation de Marmoutier, dans le BasMaine, en Anjou, par exemple et la publication récente de la thèse de

1. GANTIER, Odile, "Recherches sur les possessions et les prieurés de l'abbaye de Marmoutier du Xe au XIII siècle ", Revue Mabillon, 53, 1963, p. 93-110 et 161-167; 54, 1964, p. $15-24,56-67$ et $125-135 ; 55,1965$, p. $32-44$ et $65-79$. On peut voir aussi Dom COTTINEAU, Répertoire topobibliographique des abbayes et prieurés de l'ancienne France, Mâcon, 1939, 2 vol.

2. Alors que la tradition archivistique est de regrouper les archives au centre départemental en rapport avec l'abbaye-mère, les archives départementales d'Indre-et-Loire ont dispersé les liasses dans les départements où étaient installés les prieurés.

3. On compte les cartulaires manceau, blésois, du Perche, du Dunois, tourangeau, vendômois. Pour la bibliographie, voir BARTHÉLEMY, Dominique, La Société dans le comté de Vendôme de l'an mil au XIV siècle, Paris, 1993. Il est nécessaire de préciser que Marmoutier avait constitué des cartulaires régionaux mais que certaines publications comme celle du cartulaire manceau sont des cartulaires factices. Cette distinction prend une grande importance aujourd'hui où une attention nouvelle est portée à la composition des cartulaires. La seule histoire générale, Dom MARTENe, Histoire de l'abbaye de Marmoutier, Chevalier, C., éd., Tours, 1874-1875, 2 vol., est évidemment aujourd'hui totalement dépassée malgré tout l'intérêt qu'elle peut présenter. 
J.-H. Foulon vient éclairer de façon novatrice le rôle réformateur de l'abbaye $^{4}$. Aussi, peut-on considérer comme légitime de se pencher sur un autre cas strictement régional : la Bretagne. Plusieurs raisons militent en faveur d'une telle enquête. D'abord, il s'agit d'une entité politique bien déterminée qui conserve une grande originalité, et la place de Marmoutier dans les relations politiques et religieuses avec la Bretagne n'a rien d'anecdotique. Ensuite, les sources subsistantes se révèlent, à l'échelle des fonds bretons, plutôt considérables. Les archives départementales d'Ille-et-Vilaine ne possèdent pas moins de 250 chartes ou notices en originaux ou copies et d'autres fonds peuvent être sollicités. La matière est abondante, d'autant plus que les copies systématiques d'A. de La Borderie offrent au chercheur une première approche commode ${ }^{5}$.

Par ailleurs, le monde des prieurés paraissait plutôt délaissé il y a quelques années alors qu'il contribue fortement à nourrir cartulaires et chartriers. Or, ces institutions jouent un rôle considérable dans la société féodale et leurs archives éclairent souvent son fonctionnement ${ }^{6}$. Pour toutes ces raisons, nous avons entrepris depuis plusieurs années à Rennes 2 une enquête systématique : plusieurs mémoires de maîtrise puis masters ont été soutenus sur la question, des thèses ont été entreprises ${ }^{7}$. C'est donc un bilan de ces approches qui est proposé ici avec ses avancées et ses domaines mal éclairés, cependant, incontestablement, le dossier de

4. Legros Sébastien, Moines et seigneurs dans le Bas-Maine. Les Prieurés bénédictins du $X^{e}$ au XIII siècle, Rennes, PUR, 2010; LAMY Claire, L'Abbaye de Marmoutier (Touraine) et ses prieurés dans l'Anjou médiéval (milieu du XIe siècle-milieu du XIII siècle, thèse de doctorat, ms, Université de Paris IV, 2009; Foulon, Jean-Hervé, Église et réforme au Moyen Âge. Papauté, milieux réformateurs et ecclésiologie dans les Pays de Loire au tournant des $X I^{e}$-XII siècles, Bruxelles, 2008. On ne peut ignorer le gros travail de BARTHÉLEMY, Dominique, La Société dans le comté de Vendôme..., op. cit., qui consacre de nombreuses pages aux prieurés de Marmoutier.

5. Pour un inventaire de ce fonds : HÉnOT Aurélien, Les Moines, le châtelain et les hommes. Le rôle politique et social des prieurés de Marmoutier près de Gahard et dans les seigneuries banales de Fougères et de Vitré, mémoire de maîtrise, Rennes 2, 1998. Les actes originaux, plusieurs dizaines, se trouvent aux Arch. dép. Ille-et-Vilaine en 6H. Les copies pour toute la Bretagne sont dans le fonds La Borderie, Arch. Dép. Ille-et-Vilaine, 1F 521 à 544. Les publications sont très incomplètes : Dom MORICE, Mémoires pour servir de preuves à l'histoire ecclésiastique et civile de Bretagne, t. I, Paris, 1747, LA BORDERIE A. de et LABIGNEVILLENEuve, Paul de, " Documents inédits sur l'histoire de la Bretagne. Chartes du prieuré de la Sainte-Trinité de Fougères ", Bulletin archéologique de l'Association bretonne, 1851, p. 178-199, 1852, p. 236-250; les chartes des prieurés du Morbihan sont dans RosENSWEIG, Louis, Cartulaire général du Morbihan, Vannes, 1895, des Côtes d'Armor dans GESLIN de Bourgogne et BARTHÉlÉMY A. de, Anciens évêchés de Bretagne, le diocèse de Saint-Brieuc, Saint-Brieuc, Paris, 1895. Pour le diocèse de Nantes, LA BORDERIE A., de, « Inventaire analytique des titres des prieurés de Marmoutier situés dans l'évêché de Nantes ", Bulletin de la Société d'histoire et d'archéologie de Nantes et de la Loire-Inférieure, t. 6, 1866, 1867, passim.

6. Piснот, Daniel, Le Village éclaté, habitat et société dans les campagnes de l'Ouest au Moyen Âge, Rennes, PUR, 2001, p. 136-142. Quelques prieurés ont fait l'objet d'études particulières qui seront citées dans les notes de cette contribution.

7. Une première synthèse a été esquissée dans : PIснот, Daniel et MAzEL, Florian (dir.), Prieurés et société au Moyen Âge, Annales de Bretagne et des Pays de l'Ouest, n ${ }^{\circ}$ spécial, t. $113,2006, \mathrm{n}^{\circ} 3$. 
Marmoutier se présente comme le plus étoffé des grandes abbayes de la région. On perçoit plus clairement les raisons qui ont provoqué les implantations pour aboutir à la construction d'un réseau particulièrement dense.

\section{Les motifs d'une implantation}

Les abbayes ligériennes, angevines ou tourangelles se sont montrées fort actives en Bretagne et leurs prieurés s'y sont implantés de façon très dense mais, incontestablement, la palme revient à l'abbaye de Marmoutier, qui installa dans le duché plus d'une trentaine de dépendances. Le chiffre demeure difficile à fixer, la notion même de prieuré étant assez, fluctuante sans compter qu'il faut prendre en considération l'évolution : regroupements, disparitions... Une relation aussi privilégiée tient à de multiples facteurs qui méritent d'être élucidés.

\section{Le choix ducal}

L'abbaye de Saint-Martin jouit d'un rayonnement incomparable, le constat n'est pas nouveau mais la réception de son influence a besoin d'une étude approfondie surtout en Bretagne, terre quelque peu éloignée. Tout d'abord, traditionnellement, le duché se tourne vers la vallée de la Loire en matière religieuse et la réforme des abbayes au XI ${ }^{\mathrm{e}}$ siècle est accomplie avec la participation de Fleury ou Saint-Florent de Saumur qui ont connu le renouveau précocement mais en se distinguant sur des points essentiels du modèle clunisien qui les a touchées au départ ${ }^{8}$. Marmoutier, réformée par Maïeul de Cluny à la fin du x $\mathrm{x}^{\mathrm{e}}$ siècle, connaît une crise au tournant de l'an Mil mais aborde le $\mathrm{XI}^{\mathrm{e}}$ avec une vigueur considérable ${ }^{9}$. Ayant à sa tête des abbés prestigieux comme Albert (1032-1063), puis Barthélémy (1063-1084), elle devient un pilier de la réforme de l'Église et ne tarde pas à étendre une influence incontestée dans un ouest de la France considérablement élargi. En effet, si elle milite bien évidemment pour une réforme des mœurs et le respect de la règle, à la différence de Cluny, dont elle s'est séparée en 998, elle demeure profondément attachée à un modèle néo carolingien qui ne remet guère en cause le pouvoir princier, ducal ou comtal car elle lui reconnaît la possibilité de mener la réforme ou tout au moins d'exercer une influence majeure ${ }^{10}$. Elle demeure donc dans l'orbite de la puissante maison de Blois qui en a d'ailleurs fait son sanctuaire familial; ce n'est que plus tard, dans le cours du $\mathrm{XI}^{\mathrm{e}}$ siècle, que cette orientation se modifie progressivement.

Se développe alors autour de Marmoutier un vaste système d'alliance avec l'aristocratie, la prière pour les morts mais, plus globalement, la créa-

8. ChÉDEVILLE, André, « La renaissance du clergé régulier ", dans : DelumEAu, Jean (dir.), Histoire du diocèse de Rennes, Paris, 1979, p. 51-53; CHÉDEVILLE, André et TONNERRE, NoëlYves, La Bretagne féodale, XI'-XIII siècle, Rennes, 1987, p. 223-226.

9. Foulon, Jean-Hervé, op. cit., p. 76-78.

10. Ibidem, chap. 1. 
tion d'un lien fort entre moines et aristocrates, l'amicitia, contribue à l'élaboration d'une vaste zone d'influence et d'un domaine particulièrement étendu et dense grâce aux très nombreuses donations dont la communauté est comblée. Biens fonciers, biens ecclésiastiques et droits seigneuriaux contribuent à ériger une véritable puissance ${ }^{11}$.

Pour cette raison, les comtes et ducs de Bretagne ne tardent pas à se tourner vers une aussi prestigieuse abbaye. La formule de Marmoutier convient bien à ces princes qui initient et dirigent la réforme religieuse, y discernant certainement le moyen d'affermir un pouvoir encore fragile ${ }^{12}$. Entre 1013 et 1022, Alain III concède à Saint-Florent de Saumur l'église et le domaine de Livré-sur-Changeon en confirmant peut-être une donation de son père, ce qui ferait remonter à la fin du $\mathrm{x}^{\mathrm{e}}$ siècle les premières relations avec les moines ${ }^{13}$. Ainsi naît le premier prieuré ligérien en Bretagne ${ }^{14}$. Très vite cependant, ses difficultés surmontées, Marmoutier s'impose. Le même Alain III, entre 1015 et 1019, autorise son fidèle Guiddenoch à donner le vieux monastère ruiné de Saint-Exupère, en Gahard aujourd'hui, pour y fonder un prieuré et installer des paysans sur un défrichement ${ }^{15}$. En fait, les donations suivantes le montrent clairement, l'initiative appartient bien au duc.

Ce choix manifeste, outre des raisons religieuses, les alliances politiques de la Bretagne. Menacée par la Normandie au nord mais surtout par l'Anjou qui ne fait aucun mystère de ses visées sur le Nantais, la famille ducale a opté depuis la seconde moitié du $\mathrm{X}^{\mathrm{e}}$ siècle pour un rapprochement avec la puissante maison de Blois, elle aussi en butte aux ambitions angevines. Des mariages, en particulier, sont venus renforcer les liens. Alain III a épousé Berthe, fille du comte Eudes II vers 1020-1030. Dans ces conditions, l'appel à Saint-Florent puis à Marmoutier, alors dans l'orbite blésoise, se conçoit facilement ${ }^{16}$.

11. On découvre un aperçu de ces donations qui aboutissent souvent à la création de prieurés dans Dom MARTENE, op. cit., t. 1.

12. ChÉdeville, André et Tonnerre, Noël-Yves, op. cit., p. 221-229; Mathieu, Etienne, L'aristocratie et l'Église en Bretagne, fin $X^{e}$-début XII siècle. L'exemple des familles ducales et de Dol-Combourg, mémoire de Master 2, Rennes 2, 2005, chap. 1, Les aristocrates et l'Église : le poids de la tradition carolingienne.

13. Gullotel, Hubert, Les Actes des ducs de Bretagne (944-1148), Thèse ms, Université de Paris, 1973, n 3; Livré, dép. Ille-et-Vilaine, canton de Liffré.

14. On trouvera une étude commode quoiqu'un peu vieillie pour chaque prieuré du diocèse de Rennes dans : Gulllotin de CoRson, chanoine, Pouillé historique de l'archevêché de Rennes, Paris-Rennes, 1880-1886, 6 vol.

15. "Ego Alanus divina ordinante misericordia comes et dux britannorum una cum fratre Heudone et matre mea Hadvisa notum fieri volumus... quoniam deprecatus est nos quidam fidelis noster nomine Guiddenoch una cum filiis suis Glef, Mainone et Guiddenoch ut monasterium quodam situm in pago Redonense quod vocantur sanctum Exuperium [...] sancto Martino Majoris Monasterii concederemus [...] ", Gulllotel, Hubert, op. cit., 19; HÉNOT, Aurélien, Les Moines, le châtelain et les hommes, op. cit., p. 15. Gahard, dép. Ille-et-Vilaine, canton de Saint-Aubin-d'Aubigné.

16. ChÉDEVILle, André et TonNERRE, Noël-Yves, op. cit., p. 23-35. 
Il est vrai que la conquête angevine perturbe ces relations. La prise de la Touraine en 1044 par Geoffroy Martel aurait pu ruiner Marmoutier. Il n'en fut rien, des liens solides perdurèrent et le prestige des abbés réformateurs contribua à maintenir les positions, sinon à les renforcer. Les ducs vont d'ailleurs poursuivre leur protection à l'égard de Marmoutier et, sans que ce soit l'une de leurs préoccupations majeures, demeurent attentifs à son égard. Ils confirment très généralement les fondations de leurs vassaux fondateurs de prieurés ou les laissent agir et accordent des dispenses de coutumes sur leurs terres, par exemple en Vendelais ${ }^{17}$. Conan II, entre 1050 et 1054, en allant chez son oncle Thibaud III, rend visite à Marmoutier et lui confirme toutes ses possessions dans le duché. Enfin, encore en 1110, le duc Alain Fergent donne, à Nantes, l'église Sainte-Croix appelée à devenir le centre d'un important prieuré dans une ville majeure de la principauté ${ }^{18}$.

\section{Le relais des grands}

Très rapidement, les moines tourangeaux s'étendent en Bretagne, multipliant les fondations. En règle générale, au moins au dire des chartes, un grand aristocrate demande à Marmoutier de venir créer un prieuré à partir d'un don initial, le plus souvent d'une église et de terres. L'aspect réformateur se trouve donc bien inclus, mais parmi bien d'autres motivations. Dans le groupe des initiateurs de l'intense vague de créations qui marque la seconde moitié du $\mathrm{XI}^{\mathrm{e}}$ siècle, se pressent les plus grands du duché. Le vicomte de Donges, Frioul, dès la moitié du siècle invite les moines sur les bords de la Loire, plus tard ce sera au tour de la famille de Porhoët/Rohan de leur faire appel pour son prieuré de Josselin en leur donnant sa chapelle castrale au début du XII ${ }^{\mathrm{e}}$ siècle ${ }^{19}$. Enfin, les Dol-Combourg, issus des vicomtes d'Alet installent Marmoutier à Combourg et le vicomte de Léon en fait autant à Morlaix ${ }^{20}$.

17. MathiEu, Etienne, op. cit., p. 48. Michel Brand'Honneur a bien montré que les ducs confirment les fondations de prieurés sur les seigneuries qu'ils veulent surveiller : Fougères, Combourg mais laissent agir les vassaux les plus sûrs comme à Vitré, BRAND'Honneur, Michel, Manoirs et châteaux dans le comté de Rennes. Habitat à motte et société chevaleresque (XI -XII s.), Rennes, PUR, 2001, p. 121-124. La concession de Conan à Marmoutier, GUILLOTEL, Hubert, op. cit., 54, la teneur de l'acte est résumée dans : MoRiN, Stéphane, Trégor, Goëlo, Penthièvre. Le pouvoir des comtes de Bretagne du Xf au XIII siècle, Rennes, 2010, p. 77.

18. LABORDERIE A. de, Inventaire analytique, t. 7, p. 157-158.

19. GUILLOTEL, Hubert, «Les origines du bourg de Donges. Une étape de la redistribution des pouvoirs ecclésiastiques et laïques, aux XI'-xII siècles ", Annales de Bretagne et des Pays de l'Ouest, t. 84, 1977, p. 541-552; BouRRIQUEN, Arnaud, Les vicomtes de Rennes et leurs descendants les Porhoët/Rohan face au pouvoir ducal (fin $X^{e}$-fin XII ${ }^{e}$ s.), Mémoire de Master 2, Université Rennes 2, 2008, p. 148.

20. GuILLOTEL, Hubert, " Combour : proto-histoire d'une seigneurie et mise en œuvre de la réforme grégorienne ", dans Keats-Rohan, K. S. B. (dir.), Familiy Trees and the Roots of Politics. The Prosopography of Britain and France from the Tenth to the Twelfth Century, Woolbridge, 1997, p. 269-298; KERNEVEZ, Patrick, « Morlaix, bourg castral : du Mons Relaxus 
Très proches de ce haut niveau aristocratique, les chefs de grandes seigneuries, les barons, sont systématiquement présents. Dès le début du $\mathrm{XI}^{\mathrm{e}}$ siècle (1015-1032), Rivallon le vicaire les installe à Marcillé-Robert avant que son petit-fils leur offre une somptueuse fondation pour douze moines, une quasi abbaye, auprès de son nouveau et puissant château de Vitré (1063$1076)^{21}$. Les Fougères agissent de même en multipliant les créations au gré de leur ascension. Main ${ }^{\mathrm{er}}$ échoue dans un premier temps à Louvigné-duDésert, mais son petit-fils, Main II, concrétise l'établissement des moines à Saint-Sauveur-des-Landes entre 1040 et 1047, nouvelle fondation au cœur des terres des Fougères dont il vise à en faire une sorte de nécropole familiale. Dans un troisième temps, le château de Fougères devenant la base du pouvoir du lignage, Raoul y installe un prieuré, la Sainte-Trinité, qui témoigne de sa nouvelle puissance ${ }^{22}$. Adoptent la même ligne de conduite, les seigneurs de Châteaubriant, du Pèllerin, de Machecoul, etc. ${ }^{23}$.

Pour certaines fondations, interviennent des personnages de moindre dimension comme ce chevalier qui donne les terres de Sougéal ${ }^{24}$. À vrai dire, Hamon, suffisamment doté pour offrir trois églises agit avec l'accord, mais plus vraisemblablement sur le conseil, de son seigneur, Main II de Fougères et avec l'aval du comte. Le processus complexe n'est donc pas lié à une décision strictement personnelle. Bien entendu, les nombreuses donations complémentaires voient les vassaux suivre leur patron et l'on ne compte pas tous ces chevaliers et petits aristocrates qui apportent, une terre, une dîme ou une portion d'église. C'est tout le milieu aristocratique que l'on retrouve parmi les bienfaiteurs et qui tient à entretenir des relations privilégiées avec les moines. Au-delà, pourtant, apparaissent un peu quelques personnages plus modestes mais dotés d'une certaine fortune. Sans doute faut-il mettre à part, (mais jusqu'à quel point?) le cas particulier des aloiers de Nort-sur-Erdre qui possèdent l'église en une association de plusieurs dizaines de personnes. Ce sont des paysans visiblement relative-

à la citadelle ", Mémoires de la société d'histoire et d'archéologie de Bretagne, t. 80, 2002, p. 5-52.

21. HÉNOT Aurélien, op. cit, p. 14-15; Pichot, Daniel, "Vitré, $\mathrm{X}^{\mathrm{e}}$-XIII ${ }^{\mathrm{e}}$ siècle, "Naissance d'une ville" ", Mémoires de la société d'histoire et d'archéologie de Bretagne, t. 84, 2006, p. 5-28, Brand'Honneur, Michel, "Origines ", dans Pichot, Daniel, LAGIER Valérie, AlLain, Gwénolé (dir.), Vitré, Histoire et patrimoine d'une ville, Paris, 2009, p. 34-35.

22. MAZEL, Florian, "Seigneurs, moines et chanoines : pouvoir local et enjeux ecclésiaux à Fougères à l'époque grégorienne (milieu XI ${ }^{\mathrm{e}}$-milieu XII ${ }^{\mathrm{e}}$ siècle) ", dans PICHOT, Daniel et MAZEL Florian, Prieurés..., op. cit., p. 105-135.

23. Les actes sont analysés dans La Borderie, A. de, Inventaire, t. 6, p. 103, t. 7, p. 184, 275; Tonnerre, Noël-Yves, "Quelques remarques sur les origines de Machecoul ", dans Laurent, Catherine, Merdrignac, Bernard, Pichot, Daniel, Mondes de l'Ouest et villes du monde. Regards sur les sociétés médiévales. Mélanges en l'honneur d'André Chédeville, Rennes, 1998, p. 155-164.

24. «Ego, Haimo, patria Brito, filius Gingomari cognomento Blocci, homo et militiae deditus [...] do [...] Et ut pleniorem obtineat haec mea donatio firmitatem, dominus meus, Maino, de cujus fevo omnia quae supradicta sunt teneo, auctorizavit, libenti animo, necnon et comes Britannorum Conanus et avunculus ejus Eudo [...] ", Arch. dép. des Côtes-d'Armor, H (prieuré de Saint-Malo de Dinan) (original), éd. Dom Morice, Preuves, t. 1, col. 410-411. 
ment aisés mais leur donation semble contrainte par l'évêque ${ }^{25}$. C'est donc surtout avec l'ensemble de l'aristocratie que des liens étroits se tissent.

Les finalités se révèlent évidemment variées et malaisées à identifier car peu exprimées et de toute façon par la plume des moines rédacteurs des actes. Le souci majeur semble bien d'établir des relations étroites avec le monde monastique dont Marmoutier incarne l'accomplissement idéal mais des retours religieux et même extra religieux sont envisagés. La prégnance du modèle princier et donc l'imitation de l'action comtale s'imposent à des grands châtelains qui prennent, à ce moment, une certaine marge de manœuvre sinon d'indépendance et le prieuré vient sacraliser leur pouvoir qui s'étend. Plus clairement, la fondation assume souvent un rôle dans l'affirmation du lignage et de sa memoria. Sans que l'on puisse parler systématiquement de nécropole familiale, certains fondateurs envisagent de se faire inhumer dans leur prieuré, c'est le cas de Frioul à Donges et Triscan, fils de Rivallon, repose à Marcillé tandis que Saint-Sauveur-des-Landes semble destiné à recevoir les tombes de la famille fondatrice ${ }^{26}$. À Fougères, une notice précise que le prieuré a été fondé à partir d'une église érigée par l'épouse du seigneur implorant la guérison de son fils atteint d'une grave maladie. Le miracle s'insère alors lourdement dans l'avenir du lignage ${ }^{27}$. De façon générale cependant, les donateurs sont associés au bénéfice de la prière des moines, là réside l'objectif premier.

S'ajoutent à ces considérations religieuses des raisons beaucoup plus pratiques qui n'excluent en rien les premières, le cas de Béré l'illustre parfaitement. Une vive procédure opposant l'abbaye de Redon et Marmoutier, et remontée à Rome, permet de comprendre l'appel aux moines tourangeaux. Vers 1030, le seigneur de Châteaubriant, Brient et sa mère Innoguent désirent fonder un prieuré et un bourg. Pour cela, ils s'adressent successivement à Saint-Sauveur de Redon, puis Saint-Melaine de Rennes mais devant les faibles résultats se tournent enfin vers l'abbaye lointaine de Marmoutier dont les capacités se révèlent nettement plus adaptées aux souhaits des fondateurs ${ }^{28}$. Sans que cela soit aussi manifeste ailleurs, les moyens et les compétences de Marmoutier sont particulièrement appréciés et l'abbaye s'est fait reconnaître une spécialité dans l'organisation des bourgs et prieurés, son incontestable savoir-faire lui vaut une flatteuse réputation. Là réside, sans doute, l'une des raisons qui lui permettent

25. LABORDERIE A. de, Inventaire ..., t. 7, p. 183. La notice rédigée par La Borderie dit : « Les aloyers de Saint-Georges requis par l'évêque et l'abbé... "

26. GuILLOTEL, Hubert, « Les origines du bourg de Donges... ", op. cit.; HÉNOT, Aurélien, op. cit., p. 13.

27. Le prieuré de Fougères fait l'objet de deux actes séparés d'un demi-siècle environ et qui donnent des versions sensiblement différentes liées au contexte des relations entre les moines et le seigneur. C'est le second acte, une notice, qui relate la guérison miraculeuse mettant en lumière le rôle divin dans la sauvegarde du lignage, MAZEL, Florian, $o p$. cit., p. 115-119.

28. MEURET, Jean-Claude, Peuplement, pouvoir et paysage sur la marche Anjou-Bretagne (des origines au Moyen Âge, Laval, 1993, p. 431. 
d'éclipser les communautés locales. Tout concourt donc à faire de cette maison une référence majeure pour l'ensemble de l'aristocratie, elle incarne en tous points l'idéal monastique et la vie chrétienne capable de mener au salut, tout en apportant un certain nombre de solutions pratiques aux châtelains qui veulent établir et manifester leur puissance. Sans doute, cela se fait-il au gré des projets monastiques car, si les actes montrent surtout comment les moines répondent aux appels des laïcs, ceux-ci ne mettent pas moins en œuvre leur propre stratégie.

\section{Stratégie monastique}

Les fondations de prieurés demeurent, en dernier ressort, une décision monastique. Les raisons qui les motivent ne sont pas toujours aisées à définir, les chartes et notices demeurant plutôt discrètes sur le sujet, d'ailleurs beaucoup d'actes initiaux se présentent comme des donations et non des créations au sens strict. Il ne faut d'ailleurs pas penser à un programme planifié visant à la construction d'un temporel soigneusement élaboré. Cependant, un certain nombre d'idées et de principes semblent guider l'action monastique et, sur certains points, se dégagent des positions sensiblement différentes des autres abbayes, en particulier Saint-Florent de Saumur que l'on retrouve sur les mêmes voies ${ }^{29}$.

La création d'un prieuré répond au déroulement d'un processus complexe et souvent étalé dans le temps ${ }^{30}$. La donation initiale n'entraîne pas ipso facto l'installation des moines, et souvent, une notice pourtant détaillée n'entraîne pas de certitude sur la réalité de la présence monastique. Il faut que le temporel réuni atteigne une masse critique pour que l'on juge possible le développement d'une obédience. À Louvigné-de-Bais, la première donation n'eut pas de suite et la seconde laisse perplexe ${ }^{31}$. À Vitré, grosse fondation certes, trois étapes sont bien mises en évidence. Dans un premier temps, un accord public est scellé à Vitré entre Robert, maître du château, et le moine Urvodius, par la remise d'un couteau, puis allant à Rome, le seigneur s'arrête à Marmoutier et redonne un couteau à l'abbé en présence du chapitre puis remet le don sur l'autel. Dans un troisième temps, intervient la consécration du cimetière qui vaut inauguration. L'abbé Barthélémy est venu en personne ainsi que l'évêque de Rennes, Main, qui cautionne de son autorité la donation et bénit solennellement le cimetière ${ }^{32}$. Les choses

29. Beaumon, Jérôme, Les cadres d'implantation des prieurés de Saint-Florent de Saumur dans le Rennais (XI'-XII siècle). Éléments pour une approche comparative avec les prieurés de Marmoutier, mémoire de Master 2, Rennes 2, 2008.

30. Ce processus a été longuement étudié pour le Bas-Maine dans LEgRos Sébastien, op. cit., particulièrement chap. 4, "Le processus fondateur " et 5, "Abbayes, prieurés et programme de fondations".

31. L'existence de ce prieuré est mise en doute pas HÉNOT, Aurélien, op. cit., p. 24.

32. " [...] de his omnibus feci prius donum apud Vitriaco in magno conventu hominum tam nobilium quam innobilium, revestiens inde per unum cultellum fratrem nomine Urvodium [...] Postea cum Romam pergerem feci iterum donum in capitulo Majoris Monasterii sicut prius per unum cultellum que primum abbati Bartholomeo tradidi, deinde super altare posui 
ne sont pas toujours aussi détaillées mais l'abbé se déplace très souvent pour la remise du don et consacre ainsi la naissance de la future obédience, à moins qu'il ne vienne confirmer dans d'autres cas une institution qui se révèle viable. Cette présence abbatiale va de soi pour l'installation de prieurés sur les grands sites castraux et contribue à instaurer des relations privilégiées avec la haute aristocratie, ainsi l'abbé apporte lui-même les reliques destinées à la chapelle de Josselin après les avoir prélevées solennellement dans le reliquaire de Marmoutier ${ }^{33}$.

Les relations familiales interfèrent fortement dans ce jeu et les aristocrates devenus moines nourrissent les relations avec leur lignage et provoquent ou conseillent de nouvelles donations. Nombre de moines des prieurés ont un lien avec les familles fondatrices et le cas du prieur de Saint-Martin de Combourg est exemplaire. Membre d'un lignage, lié à deux autres qui ont fourni de multiples recrutements, et non des moindres, aux abbayes de Saint-Florent et de Marmoutier, le jeune Garin de Lanrigan se forme auprès du prieur de Fougères, Guillaume, son oncle, avant de prendre en main le prieuré de Combourg en 1095. Il manifeste un dynamisme qui modifie localement les jeux monastiques. Les donations affluent au bénéfice de Marmoutier alors que jusque là, Saint-Florent obtenait la première place, sans doute à la faveur de l'abbatiat de Guillaume de Dol $^{34}$. L'oncle qui appartient au lignage des Le Chat, bien établi dans la région de Combourg, devient abbé de Marmoutier de 1105 à $1124^{35}$. Il est inutile de souligner l'intérêt de cette élection pour l'influence de l'abbaye dans la région. Il n'en demeure pas moins quelque peu paradoxal de voir accéder à des postes clefs des membres de familles notoirement simoniaques et nicolaïtes et qui pourtant sont porteurs de renouveau ${ }^{36}$.

Le Grand Monastère accorde beaucoup d'importance aux relations avec les évêques, indispensables soutiens à son action. De toute évidence, les premières donations vont vers une abbaye réformée même si elle ne se soucie pas toujours de mettre en application ses principes sur le terrain. Très nettement, les nouveautés se dessinent autour de 1050 et l'on peut voir en Airard, évêque de Nantes, le principal organisateur mais sans doute

[...] Facta est haec donatio per assensum et auctoritatem domini Mainoni Redonensis episcopi quando ipse et Bartholomeus [...] ", Dom Morice, Preuves, col. 424-425.

33. " [...] aperuit ipse domnus abbas Willelmus capsas in quibus reliquie continentur in monasterio nostro et accepit inde preciosissima immo inestimabilia pignora sanctarum reliquiarum [...] Has igitur supradictas reliquias diligentissime collacatas transtulit domnus abbas in Britanniam et posuit eas in supradictam cellam [...] ", RosENZWEIG, L., Cartulaire général du Morbihan, $\mathrm{n}^{\circ} 185$.

34. Guillotel, Hubert, "Combourg... ", op. cit., p. 289.

35. "Guillaume le Chat ne faisait qu'un avec le futur abbé de Marmoutier ", ibidem, p. 281.

36. Hubert Guillotel a montré comment trois familles de la région de Combourg, particulièrement engagées dans les carrières cléricales, ont fourni des abbés à Saint-Florent de Saumur et Marmoutier. Si les moines recrutés furent des partisans de la réforme, les abbayes durent parfois composer et ainsi Marmoutier confia à un fils de prêtre la direction du prieuré de Combourg, Guillotel, Hubert, " Combour... ", op. cit., p. 285. 
pas le seul. Ce cardinal envoyé tout exprès par le pape Léon IX, proclame sa volonté d'imposer la réforme dans un diocèse passablement éprouvé. Significativement, c'est dans un acte accordé à Marmoutier qu'il définit son action après avoir rappelé les décrets du pape condamnant la possession de biens ecclésiastiques par les laïcs sous peine d'excommunication. Le seigneur du Pellerin donne alors six églises que l'évêque propose de transmettre à Marmoutier " [...] Quas ego funditus, sicut ille michi dimiserat omnes sancto Martino et monachis Majoris Monasterii dedi et propter eorum preconabilem in Domini servitio fervorem et ut mei meorumque decessorum atque successorum atque mihi commisi gregis habere dignarentur in suis benefactis memoriam ${ }^{37}$. "L'évêque réécrit un peu l'histoire car la donation avait été préalablement faite à Marmoutier sans son intermédiaire. Il veut affirmer l'autorité épiscopale en faisant cela et en imposant le paiement aux moines d'un cens en or pour l'église de Béré qu'il leur accorde. Obligé rapidement à la démission en raison de son intransigeance, il est remplacé par Quiriac qui continuera la même politique. Ce n'est donc pas sans raison que l'on voit se multiplier les fondations durant la seconde moitié du $\mathrm{XI}^{\mathrm{e}}$ siècle, l'abbaye a partie liée avec les réformateurs ${ }^{38}$.

Les mêmes rapports semblent en effet s'être établis avec les évêques de Rennes : Sylvestre de La Guerche (1076-1093) dont l'archidiacre n'est autre que Robert d'Arbrissel, puis Marbode. Il en va de même à Alet mais la suppression du prieuré lors du transfert du siège épiscopal à Saint-Malo provoque un dur conflit (1144-1146) ${ }^{39}$. Les nouveaux prieurés se multiplient avec pour corollaire les dons massifs d'églises et de cimetières. La quasitotalité des 28 ou 29 églises détenues par Marmoutier dans le diocèse de Rennes est acquise dans la seconde moitié du XI ${ }^{\mathrm{e}}$ siècle. Ses efforts placent l'abbaye, dès le xII ${ }^{\mathrm{e}}$ siècle, à la tête d'une masse considérable d'églises paroissiales et son patronage établi sur environ 98 , lui octroie la première place en Bretagne ${ }^{40}$.

37. «Et moi j’ai tout donné, intégralement comme il me les avait laissé, à saint Martin et aux moines de Marmoutier, tant à cause de leur ferveur digne d'éloge dans le service du Seigneur que pour qu'ils daignent garder en leurs bienfaits mémoire de moi, de mes prédécesseurs et de mes successeurs ainsi que du troupeau qui m'est confié... ", idem, " La pratique du cens épiscopal dans l'évêché de Nantes. Un aspect de la réforme ecclésiastique en Bretagne dans la seconde moitié du xI ${ }^{\mathrm{e}}$ siècle ", Le Moyen Âge, t. 80, 1974, p. 5-49, texte latin p. 34-40. Le texte traduit est publié dans Brunel, Ghislain et Lalou, Élisabeth (dir.), Sources d'histoires médiévale, IX ${ }^{\mathrm{e}}$-milieu du XIV ${ }^{\mathrm{e}}$ siècle, Paris, 1992, p. 152-154.

38. Foulon, Jean-Hervé, op. cit., p. 328-335.

39. ChÉDEVILLE, André, "Le temps des réformes... ", dans DeluMEAu, Jean, Histoire des diocèses..., op. cit., p. 59-60; ChÉDEVILLE, André et TonNERRE, Noël-Yves, La Bretagne féodale, op. cit., p. 259, Guillotel, Hubert, "Les évêques d'Alet du IX au milieu du XII ${ }^{\mathrm{e}}$ siècle ", Annales de la société d'histoire et d'archéologie de Saint-Malo, 1979, p. 251-266.

40. Devailly, Guy, "Une enquête en cours. L'application de la réforme grégorienne en Bretagne ", Annales de Bretagne et des Pays de l'Ouest, t. 75, 1968, p. 293-316; idem, "Religion et politique en Bretagne aux XI ${ }^{\mathrm{e}}$ et XII ${ }^{\mathrm{e}}$ siècles : le cas des patronats paroissiaux ", dans Laurent, Catherine, Merdrignac, Bernard, Pichot, Daniel (dir.), Mondes de l'Ouest et villes du monde. Regards sur les sociétés médiévales. Mélanges en l'honneur d'André Chédeville, p. 325-337. Le chiffre est pris dans DuFIEF, André, Les Cisterciens en Bretagne, 
Pour autant, des compromis demeurent possibles pour la sauvegarde des intérêts monastiques. L'exemple le plus criant se situe à Fougères. Dans un affrontement avec les chanoines de la collégiale, l'abbaye proposa de prêter une forte somme d'argent au seigneur pour demeurer en possession d'une église, ce qui lui valut les foudres épiscopales de Marbode ${ }^{41}$. Néanmoins, c'est un bilan imposant que l'on peut dresser quand on examine la part bretonne de l'empire de Marmoutier. L'abbaye a considérablement étendu son influence sur le duché y apportant le renouveau monastique et obtenant un considérable prestige auprès de l'aristocratie. En tous points, elle éclipse les autres maisons bretonnes aussi bien qu'extérieures.

\section{L'empire de Marmoutier en Bretagne}

L'ampleur de l'implantation de Marmoutier dans le duché ne constitue pas vraiment une découverte, pourtant l'analyse précise demeure à effectuer. La réalité prieurale n'a guère fait l'objet que d'études ponctuelles, sinon monographiques souvent anciennes et une vision globale de l'action des moines tourangeaux devrait apporter de précieuses conclusions pour la compréhension des rapports entre religieux et laïcs dans le duché ${ }^{42}$. Un examen chronologique des étapes d'implantation ainsi qu'une analyse de son appréhension de l'espace breton apportent une première série d'enseignements sur les objectifs et les pratiques de cette abbaye conquérante mais il faut ensuite essayer de comprendre le système du prieuré qui s'organise et innerve la vie religieuse, certes, mais aussi largement, toute la société. Finalement se dessine une façon originale de faire jouer des facteurs classiques qui caractérise l'action du Grand Monastère.

\section{Les prieurés dans l'espace et dans le temps}

Le rythme d'installation des prieurés, donnée essentielle pour notre enquête, suscite de nombreuses difficultés liées à la documentation. Le flou des datations et surtout les incertitudes sur le moment de l'arrivée des moines rendent l'opération un incertaine, cependant des orientations générales se dégagent qui résultent du jeu des facteurs évoqués précédemment et s'inscrivent plus globalement dans le contexte à la fois de l'histoire régionale et de la réforme qui traverse l'Église entière ${ }^{43}$. Marmoutier fait l'objet des faveurs bretonnes, très tôt, dès le début du XI ${ }^{\mathrm{e}}$ siècle. Sous l'im-

$X{ }^{e}$-XIII ${ }^{e}$., Rennes, 1997, p. 197-201. Un tel chiffre donne une bonne idée de l'ampleur de l'influence de Marmoutier mais peut être discuté dans le détail car il est difficile de faire un point exact.

41. MAZEL, Florian, "Seigneurs, moines et chanoines... ", op. cit.

42. Les études anciennes sur les prieurés ne manquent pas mais souffrent de lacunes : Guillotin de Corson, Pouillé historique..., op. cit., offre un bon exemple des apports utiles et des limites de ce genre de travail. On trouvera des éléments récents sur Marmoutier dans Pichot, Daniel et MAZEL, Florian, op. cit.

43. Foulon, Jean-Hervé, op. cit., p. 68-88. 
pulsion du duc, apparaît le puissant prieuré de Gahard (1015-1019) et les deux familles en pleine ascension des futurs seigneurs de Fougères et Vitré installent les moines à Saint-Sauveur des Landes (1040-1047) et MarcilléRobert (1015-1032) ${ }^{44}$. La donation de Sougéal, confirmée par Main II date de 1040-1047 sans que l'on sache si les moines viennent à ce moment ${ }^{45}$. Sans doute grâce à l'impulsion comtale et à une certaine éclipse de SaintFlorent, le développement de la présence de Marmoutier se confirme mais sans trop grande vigueur ${ }^{46}$. Dans cette première moitié du $\mathrm{Xl}^{\mathrm{e}}$ siècle, les moines possèdent 4 ou 5 maisons dans le diocèse de Rennes mais dans les années 40, un mouvement s'amorce aussi dans celui de Nantes avec Béré et Champtoceaux ${ }^{47}$.

Commence un peu avant 1050 une seconde étape qui s'accélère surtout vers 1060. Elle se caractérise par une percée dans le diocèse de Nantes avec les fondations de Donges, Le Pèllerin, Varades, en lien visiblement avec l'arrivée de l'évêque réformateur Airard, et en s'appuyant sur de puissants aristocrates comme le vicomte Frioul. Il faut constater, en même temps, que les difficultés politiques se sont quelque peu aplanies, ce qui favorise un tel mouvement ${ }^{48}$. Les implantations initiales suivent les péripéties des histoires familiales. Le développement de la puissance châtelaines oblige à de nouvelles fondations. Les successeurs de Rivallon le Vicaire quittent Marcillé pour Vitré et ceux de Main vont à Fougères, ce qui provoque deux naissances de prieurés. Vient alors une période faste de créations le plus souvent castrales, l'abbaye confirmant délibérément son alliance avec les grands châtelains : Fougères, Vitré, Combourg... La vague s'amplifie à la fin du siècle avec des fondations plus modestes comme Pontchâteau et peut-être Saint-Ouen-la Rouërie ${ }^{49}$. En 1083, l'installation à Lamballe amorce une progression vers l'ouest et une prise de contact avec le lignage des Eudonides par l'intermédiaire de Geoffroy Boterel ${ }^{\mathrm{er}}{ }^{50}$. En cette période faste s'établissent 12 dépendances et souvent les plus importantes comme Vitré, Tinténiac, Fougères ${ }^{51}$...

44. Pichot, Daniel, "Prieurés et société... ", op. cit., Mazel, Florian, " Pouvoir local... ", op. cit.; HÉNOT, Aurélien, op. cit., p. 15, MEuRET, Jean-Claude, op. cit., p. 317.

45. GullLotel, Hubert, "Combour... ", op. cit., p. 287.

46. Cette concurrence est bien analysée par Guillotel, Hubert, "Combour... ", op. cit. et BEAUMON, Jérôme, "Les cadres d'implantation... ", op. cit., p. 104-111.

47. Gahard, Marcillé, Saint-Sauveur-des-Landes, sont sûrs. Des doutes pèsent sur l'existence de Louvigné-du Désert. Seul Sougéal serait à ajouter en raison d'une dotation initiale vers 1040-1047.

48. LABORDERIE, A. de, "Inventaire..., ", op. cit.,Dufief, André, op. cit., Gullototel, Hubert, "Le cens épiscopal...", op. cit.

49. LABORDERIE, A. de, "Inventaire. ". À Saint-Ouen, un prieuré semble exister à la fin du $\mathrm{Xl}^{\mathrm{e}}$ siècle, GulLLoTEL, Hubert, "Combour... ", op. cit., p. 290.

50. Morin, Stéphane, Trégor, Goëllo, Penthièvre, le pouvoir des comtes de Bretagne du XI au XIII siècle, Rennes, 2010, p. 304-305.

51. Voir carte par CHÉDEviLLE, André dans : LEBRun, François (dir.), L'Ille-et-Vilaine, des origines à nos jours, Saint-Jean d'Angély, 1984, p. 102. 
Dans les trois premières décennies du XII ${ }^{\mathrm{e}}$ siècle, l'élan ne connaît qu'un affaiblissement tout relatif, on compte encore une dizaine d'installations mais qui dénotent un caractère particulier. Il ne s'agit pas d'abord de densifier la trame existante même si l'arrivée à Nantes en 1106 prend valeur de symbole mais plutôt de conquérir de nouveaux espaces nettement plus éloignés vers l'ouest. Il faut ajouter Machecoul pour ce diocèse mais mais Saint-Malo l'emporte avec cinq maisons : Saint-Malo même, Josselin, Dinan, Iffendic et Bécherel. Dans les diocèses plus lointains, celui de Saint-Brieuc adjoint Jugon à Lamballe et celui de Vannes accueille alors deux maisons : Rohan et Malestroit ${ }^{52}$. Celui de Saint-Pol-de-Léon voit naître le prieuré de Morlaix (1128) et, dès 1118, l'évêque de Quimper, Robert, avait installé une modeste obédience sur l'île Tristan, devant Douarnenez ${ }^{53}$. Il faut enfin noter le cas très particulier, dans le diocèse de Dol, du prieuré de Léhon d'une importance quasi abbatiale, dépendance de Saint-Magloire de Paris qui échoit à Marmoutier, en 1181, à la faveur de débats compliqués ${ }^{54}$. Après 1130 , l'ère des fondations s'achève, cela va d'ailleurs de pair avec un net ralentissement général des dons.

Cette brève enquête chronologique éclaire notre compréhension du réseau monastique dans l'espace breton. La présence de Marmoutier se fait particulièrement dense dans les diocèses de haute Bretagne : dix prieurés dans le diocèse de Nantes (en comptant Liré en Anjou mais du diocèse de Nantes), huit ou neuf dans celui de Rennes et six dans celui de SaintMalo. Bien sûr, tous n'ont pas la même importance mais ils forment un maillage serré et s'appuient sur des pôles forts, surtout dans le diocèse de Rennes où l'action monastique rayonne à partir des maisons de Gahard, Fougères, Vitré. Dans le Nantais, les fondations sans doute un peu moins importantes conservent une densité soutenue. La carte met en évidence plusieurs traits caractéristiques. Marmoutier affiche une spécialité et même une préférence pour les sites castraux et opère un choix délibéré pour les grandes familles châtelaines. Elle privilégie une expansion à partir de forts centres de seigneuries, et non selon un mouvement de capillarité qui aurait plutôt la préférence de Saint-Florent de Saumur ${ }^{55}$. En même temps, transparaît un net intérêt pour les bords de Loire, la facilité des communications avec l'abbaye mère et l'activité commerciale sur le fleuve suffisent à rendre compte d'un tel choix ${ }^{56}$. Il faut aussi noter la multiplication des prieurés érigés en des sites politiquement sensibles sur la frontière entre Bretagne et Anjou : Champtoceaux, Varades, Liré balancent entre deux influences,

52. Morin, Stéphane, op. cit., p. 300-305; RosenzWEIG, Louis, Cartulaire général..., op. cit.

53. Kernevez, Patrick, "Morlaix, bourg castral : du Mont Relaxus à la citadelle ", Société d'Histoire et d'Archéologie de Bretagne, t. 80, 2002, p. 5-52; QuAGHEBEur, Joëlle, La Cornouaille du IX au XII s., p. 322.

54. Barral Y Altet, Xavier (dir.), Dinan, au Moyen Âge, Le Pays de Dinan, 1986. Le temporel de Léhon important s'étend assez loin vers l'ouest.

55. Guillotel, Hubert, "Combour... ", op. cit.; BEAumon, Jérôme, op. cit., p. 65-68.

56. Marmoutier obtient des exemptions de péage à Ancenis, LAMY, Claire, p. 411-412. 
et cristallisent les conflits ${ }^{57}$. Faut-il y voit l'influence angevine? Sans doute mais aussi la tendance classique à fixer les frontières par une politique de sacralisation. Enfin, si les sites châtelains focalisent l'intérêt, les villes épiscopales demeurent largement oubliées, seule Nantes est représentée, le prieuré de Saint-Malo quant à lui n'a qu'une brève existence ${ }^{58}$.

Au-delà, vers la basse Bretagne le semis se fait beaucoup moins dense et beaucoup plus tardif mais la spécificité s'affiche encore plus clairement. Les diocèses occidentaux demeurent peu représentés : un ou deux prieurés pas plus. Par contre, le choix castral ne se dément pas, seule la modeste fondation de l'île Tristan apparaît différente. Cette distorsion entre l'est et l'ouest trouve incontestablement son explication dans la distance, il faudrait sans doute invoquer aussi les questions de langue, la partie celtophone pose problème mais surtout le rythme de la réforme doit aussi être pris en compte. Si cette dernière commence fort tôt en haute Bretagne, ce n'est pas le cas à l'ouest et, quand cela se produit tardivement, l'âge des prieurés est révolu ${ }^{59}$. Cependant, avec 31 ou 32 fondations, Marmoutier a installé le premier réseau monastique en Bretagne, manifestant, son prestige et sa primauté ${ }^{60}$.

\section{Le développement de l'institution prieurale}

L'existence du prieuré apparaît, au XI ${ }^{\mathrm{e}}$ siècle, comme une nouveauté à la réalité fluctuante et qui ne se précise que peu à peu. Le haut Moyen Âge n'avait pas ignoré les domaines dépendants des abbayes mais leur fonctionnement nous échappe. Les nombreuses dépendances créées par la suite deviennent des institutions essentielles du réseau monastique bénédictin éminemment intégrées dans la société féodale ${ }^{61}$.

La grosse trentaine de fondations de Marmoutier en Bretagne présente évidemment des caractères différents et l'importance respective de chacune demeure variable. La fondation type comme Vitré, Fougères ou Gahard repose sur une "restitution " ou un transfert d'église, souvent une paroissiale mais pas obligatoirement ${ }^{62}$. Ce peut être une chapelle ancienne comme à Béré, castrale comme à Combourg et sans doute Vitré, Josselin... Cette église devient généralement la chapelle du prieuré. Si Gahard, la pre-

57. LAMY, Claire, op. cit. Il faudrait ajouter, le prieuré de Carbay en Anjou, certes, mais fondé pour le refuge du prieuré de Marcillé chassé par les guerres. Liré situé dans le comté d'Anjou dépend du diocèse de Nantes.

58. ChÉDEVILle, André et TonNERre, Noël-Yves, op. cit., p. 259.

59. Gulllotel, Hubert, "Le cens épiscopal... ", op. cit., Devallly, Guy, op. cit.

60. L'hésitation porte sur l'existence ou non d'un prieuré à Louvigné-de-Bais. Il est évident que ce chiffre demeure approximatif dans la mesure où il n'est pas toujours facile de déterminer la présence des moines. Par ailleurs, ce chiffre est un maximum et il faut noter les disparitions comme Saint-Malo ou les rattachements comme Sougéal à Dinan.

61. Pichoт, Daniel, "prieurés et société... ", op. cit.

62. Pour l'étude de quelques cas : HÉNOT Aurélien, op. cit., chap. 2 ; MAZEL, Florian, "Seigneurs, moines... ", op. cit., Gulllotel, Hubert, " Les origines du bourg de Donges... ", op. cit. 
mière création, échappe à la règle, les grandes fondations seront majoritairement castrales.

La puissance des obédiences demeure liée à l'importance du temporel associé. L'étude, pas toujours possible, bénéficie cependant de quelques dossiers étoffés. Gahard, Vitré ou Fougères évoqués précédemment possèdent de beaux domaines fonciers : labours, prairies et droits forestiers, tant pour le bois que pour l'élevage. S'ajoutent droits seigneuriaux divers, moulins et exemptions de coutumes sur les hommes au bénéfice des moines ${ }^{63}$. À Donges, les terres comprennent beaucoup de territoires en prairie sur les marais de la Brière et de la basse-Loire destinant l'élevage destiné à une réelle ampleur et Geoffroy Boterel fait une concession majeure pour la création du prieuré de Lamballe, accumulant terres, prés, moulin et droit de faire des moulins, pêcheries, droits forestiers, marchés ${ }^{64}$... Ce temporel s'enrichit ensuite de donations de la famille fondatrice ou de ses vassaux et connaît éventuellement un large développement. À Marcillé, le moine Albert déploie une grande activité et reçoit des dons visiblement sollicités complétés par des achats ${ }^{65}$.

Au gré des donations, certains prieurés s'étendent sur plusieurs paroisses et leur emprise peut s'établir sur plus de $10 \mathrm{~km}$ comme à SaintSauveur-des-Landes ou Gahard. Ces donations complémentaires apportent massivement des églises paroissiales avec leurs revenus, des cimetières et plus parcimonieusement des dîmes, ce qui place Marmoutier à la tête d'un patrimoine religieux des plus importants, les 98 églises évoquées précédemment.

Enfin, dans nombre de cas et de façon systématique dans les prieurés castraux, les moines établissent un bourg, ce qui leur apporte un nombre appréciable de bourgeois dépendants avec les coutumes afférentes ${ }^{66}$. Cela va de pair avec les droits économiques liés à l'artisanat et aux échanges. Le marché et la foire, dont les revenus sont partagés ou non, occupent une place de choix ainsi que les péages; les implantations sur la Loire sont significatives à cet égard. Le prieuré se présente donc comme une véritable petite seigneurie et c'est bien ainsi que les moines le prennent en compte ${ }^{67}$.

63. "[...] ut nec comes nec ullus ex vicariis ejusdem regionis nec alia prorsus aliqua persona praeter ipsum habere visa sit ullam dominationem in hominibus ipsius parrochiae distrigendis, nec annonaticum, nec vaccaticum, nec ullam redhibitionem, nec vel ipsum bannum [...] De silva ad excolendum distribuere ipsi monachi in quantum voluerint liberam habeant facultatem, de suis villanorumque suorum porcis pasnaticum sibi tantummodo vindicantes [...]", Dom Morice, Preuves, t. 1, col. 360, cet exemple pris dans l'acte de donation de Gahard donne un aperçu du type de donation, le défrichement de la forêt demeure quand même plutôt rare.

64. Gulllotel, Hubert, " Les origines du bourg de Donges... ", op. cit.; Gallia Christiana, t. XIV, instrumenta, col. 261-262.

65. HÉNOT, Aurélien, op. cit., p. 136-138.

66. MiYAmATSU, Hironori, Bourgs et bourgeois dans l'ouest de la France (XI'-XIII siècle), Thèse de $3^{\mathrm{e}}$ cycle, Université de Rennes 2, 1986, 2 vol.

67. ChÉDEVILlE, André, "Étude de la mise en valeur et du peuplement du Maine au $\mathrm{XI}^{\mathrm{e}}$ siècle d'après les documents de l'abbaye Saint-Vincent du Mans ", Annales de Bretagne, 
Toutes ces dépendances demeurent quand même la résidence d'une communauté religieuse, restreinte, certes, mais réelle. Vitré, cas exceptionnel, est prévu pour douze moines, ce qui ne fut pas respecté et traduit l'ambition des Vitré ou peut-être la reprise d'un vieux monastère ruiné du haut Moyen $\widehat{A} g \mathrm{e}^{68}$. Sinon, les prieurés comptent trois ou quatre moines, pas plus et le plus souvent moins, le chiffre de deux étant sans doute le plus fréquent. On en identifie quatre à Combourg mais un seul à Sougéal. Ces inégalités se traduisent par des différences dans l'organisation de ces institutions.

Peu à peu, en effet, le système se structure. Les termes les plus divers désignent au départ ces fondations avec une préférence à Marmoutier pour obedientia qui dit clairement le statut de dépendances mais on trouve aussi, cella, domus, monasteriolum ${ }^{69}$. Le terme de prioratus, comme ailleurs, arrive tardivement. Après une timide émergence à la fin du $\mathrm{XII}^{\mathrm{e}}$ siècle, il ne se diffuse vraiment qu'au cours du XIII ${ }^{\mathrm{e}}$ siècle, montrant l'arrivée de l'institution à maturité ${ }^{70}$. Toutes les fondations, cependant, ne bénéficient pas de cette appellation. Y eut-il une distinction entre les différentes maisons, seules les plus importantes, les mieux structurées pouvant accéder à cette dénomination?

Certaines, il est vrai, acquièrent une influence ou même une autorité supérieure. Les fondations d'une même famille semblent vivre sous la coupe de la dernière fondée en lien avec le château majeur. Marcillé évolue dans l'orbite de Vitré et les deux (ou trois?) fondations des Fougère sont placées sous la coupe du prieur de la Trinité de Fougères ${ }^{71}$. Jugon ne possède pas la dimension de Lamballe ou Dinan, sans parler de SaintMagloire de Léon. Logiquement, des reclassements s'opèrent si nécessaire, surtout au cours du XIII ${ }^{\mathrm{e}}$ siècle. Le prieuré modeste de Sougéal est rattaché à Dinan et celui de Saint-Malo disparaît dans des conditions exceptionnelles, le transfert de la cathédrale d'Alet à Saint-Malo entraîne sa dissolution malgré les plaintes des moines auxquelles Rome passe outre ${ }^{72}$.

Un responsable se dégage, prévôt d'abord, avant que le titre de prieur ne commence à apparaître vers $1080-1090$ pour se généraliser au XII siècle $^{73}$. Les prieurs assument un rôle important, d'abord dans la gestion de leur maison, d'autant plus que l'éloignement contraint à leur laisser une

1960, p. 209-225.

68. Suggestion de Michel Brand'Honneur, Ріснот, Daniel (dir.), Vitré, histoire..., op. cit., p. 32 .

69. BEAUMON, Jérôme, op. cit., p. 112-113; LABORDERIE A. de, «Inventaire... ", op. cit.

70. Pichot, Daniel, « Prieurés et société ", op. cit., p. 21; BEAUMON, Jérôme, op. cit., p. 113-

115; LeGRos Sébastien, Moines et seigneurs..., op. cit., p. 93-98.

71. HÉNoT, Aurélien, op. cit., p. 25-26, 53; MAZEL, Florian, " Pouvoir local... ", op. cit.

72. Sougéal : Guillotin DE CoRson, op. cit, t. 3, prieurés de Marmoutier; Saint-Malo : le prieuré est fondé en 1108 quand l'évêque d'Alet donne l'église Saint-Malo. Quand Jean de Châtillon transfère le siège épiscopal à Saint-Malo, il reprend l'église et le prieuré disparaît (1146-1152).

73. HÉNOT, Aurélien, op. cit.; Pıснот, Daniel, « Prieurés et société... », op. cit., p. 21. 
certaine autonomie. Ils gèrent leur temporel et les actes les citent comme initiateurs, Rivallon, prieur de Vitré passe un accord pour fonder le village de défrichement de Mondevert avec le seigneur de Laval (1080-1093) et le cartulaire de Combourg dévoile le dynamisme de Garin ${ }^{74}$. Les archives semblent témoigner de ce mode de fonctionnement, on retrouve pour certains prieurés comme Saint-Sauveur-des-Landes de petites pancartes regroupant cinq à six notices notant soigneusement l'évolution des biens ${ }^{75}$. Le lien avec l'abbaye mère ne se distend pourtant pas. On ignore encore ce que reverse le prieuré, les comptes ne faisant leur apparition qu'à la fin du Moyen Âge, mais dès les débuts, l'abbé maintient l'unité de l'ensemble monastique et veille à sa bonne marche. Sa présence, fréquente, s'avère systématique pour les fondations d'importance, Barthélemy préside à l'inauguration de Sainte-Croix de Vitré, Bernard reçoit le don de Lamballe à Dol, mais ces cérémonies majeures ne constituent pas des occasions uniques. À la fin du XI ${ }^{\mathrm{e}}$ siècle, l'abbé Barthélémy visite ses prieurés : "descenderet enim aliquando idem venerabilis abbas in Brittaniam causa visitandi domos quos habebat in Brittaniae partibus et hospitandi gratia venit Combunium ${ }^{76}$. "

Au début du XII ${ }^{\mathrm{e}}$ siècle, l'abbé Guillaume se fait accompagner pour ses voyages en Bretagne par les principaux prieurs avec en bonne place Geoffroy de Lanrigan, responsable de Combourg mais aussi son neveu et visiblement conseiller pour les affaires bretonnes ${ }^{77}$. On ne peut donc pas parler de structuration de type " administratif " mais d'un lien fort, entretenu soigneusement, reposant d'abord sur des liens personnels. L'abbé joue un rôle fédérateur; fréquemment en chemin, il soude l'Église du Grand Monastère aux yeux de ses moines et de toute la société.

\section{Marmoutier et la société bretonne}

Le contemptus mundi monastique n'entraîne aucunement une mise à l'écart de la société, bien au contraire. Le statut de moine bénédictin est devenu un modèle d'accomplissement chrétien et les prieurés assurent une présence monastique démultipliée et pour Marmoutier dont le prestige prime dans tout l'Ouest, l'influence pèse très lourd. Les implications sociales multiples prennent cependant des caractères un peu particuliers pour l'abbaye tourangelle ${ }^{78}$.

La question spirituelle se place au cœur du phénomène d'implantation prieurale comme le souligne J.-H. Foulon : " Les multiples prieurés, essaimés sur tout l'espace permettaient une bonne administration temporelle

74. Meuret, Jean-Claude, Peuplement..., p. 497; Gulllotel, Hubert, "Combour... ", op. cit., p. 290.

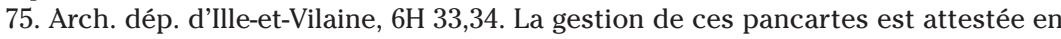
Anjou et très bien mise en lumière par Claire LAMY, op. cit.

76. Dom Morice, Preuves, c. 491.

77. Gullootel, Hubert, " Combour... ", op. cit., p. 290.

78. Les thèmes généraux sont largement développés dans : Pichot, Daniel et MAZEL, Florian, Prieurés et société, op. cit. 
et un réel rayonnement spirituel ${ }^{79}$. " Une notice de Combourg se montre particulièrement révélatrice de la place dominante du modèle monastique : une donation d'églises intervient après la guérison par l'abbé des deux fils du donateur. Barthélémy guérit d'un baiser un lépreux et change l'eau en vin, l'assimilation au Christ, évidente par ces miracles, le place très au-dessus de l'humanité ordinaire ${ }^{80}$. Un tel prestige rejaillit sur le recrutement pour lequel une enquête précise demeure largement à effectuer; les textes laissent l'impression d'une forte attraction de l'abbaye dans le milieu aristocratique où certains obtiennent l'autorisation de devenir moine ${ }^{81}$. On ignore cependant dans quelle mesure il y a passage à l'acte. Les adultes convertis ne manquent pas mais les familles tiennent à placer nombre de leurs membres dans la vie monastique : la carrière de l'abbé Guillaume et de son neveu Garin offre une bonne illustration d'un engagement familial que les notices permettent de retrouver dans bien d'autres cas. En 1093, par exemple, Adam, fils d'Amalric entre à Marmoutier et offre ses droits sur l'église ${ }^{82}$. Les lignages placent de préférence des cadets comme le prieur Rivallon, à Vitré, qui porte un nom caractéristique de la famille fondatrice ${ }^{83}$. Ces entrées dans la vie religieuse contribuent à renforcer le système des donations et surtout les liens établis avec les moines ${ }^{84}$.

Ainsi se peuplent sans doute nombre d'obédiences. Il faut, en plus, faire une place particulière à la pratique bien attestée de la conversion ad succurendum mais somme toute moins importante pour la vie des communautés. Plus largement, de nombreux donateurs, mais pas tous, accèdent à la societas beneficii. Vingt-neuf bénéficiaires y sont admis d'après une enquête limitée à quelques prieurés du diocèse de Rennes ${ }^{85}$. Le prieuré relaie donc efficacement le modèle monastique qui séduit le groupe aristocratique et renforce la symbiose entre les deux élites. Ainsi s'établit un lien fort dans une commune vue de l'au-delà. Le bénéfice s'avère considérable pour les donateurs eux-mêmes, qui par l'intercession des prières, élargissent leur capital religieux et sacré, concourant à la fois à leur Salut mais aussi à leur prestige social sur la terre.

79. Foulon, Jean-Hervé, op. cit, p. 86.

80. "Tunc venit ad eum supradictus Maino rogans eum, ut descenderet apud Gugnen visitare filios suos Haimonem et Gauterium, qui gravi tenebantur infirmitate. Descendit et signum crucis frontibus eorum imposuit, et statim obdormierunt, et post somnum integrae sanitatis restituti sunt. Quo viso et audito quod leprosum osculo sanaverat supradictus abbas, et aquam in vinum converterat [...] ", Dom Morice, Preuves, c. 491.

81. Cas de Constant à Donges, idem à Iffendic, Marcillé, Sougéal, GuILlotel, Hubert, op . cit., ; HÉnot, Aurélien, op. cit., p. 46-48; Dom Morice, Preuves, col. 410-411.

82. "[...] domnus [sic] Adam filius Amalrici, cognomento Bonelli, de Gahardo, quando factus est monachus, dedit [...] censum quemdam quem hereditario jure possidebat ein ecclesia Sancti Exuperii ", Arch. dép. d'Ille-et-Vilaine, 1 F 529. Autre cas à Marcillé, Arch. dép. d'Ille-et-Vilaine, 1 F 544/7.

83. Ibidem, col. 480-481 et Arch. dép. d'Ille-et-Vilaine, 1F 544/6.

84. HÉNOT, Aurélien, op. cit., p. 47.

85. Ibidem, p. 44-45. 
Par contre, la participation à la réforme se cantonne dans certaines limites bien ancrées dans le cadre grégorien. Marmoutier bénéficie fortement des transferts de biens ecclésiastiques encouragés par le discours réformateur et se place au premier rang pour les dons d'église. Les moines visent d'ailleurs très nettement les églises et plus particulièrement les paroissiales. Autour des grands prieurés gravitent nombre de lieux de culte, celui de Béré n'en contrôle pas moins de quinze. Les évêques encouragent notoirement le mouvement, y percevant sans doute un bénéfice pour l'encadrement paroissial ${ }^{86}$. On ne peut contester une évolution globale de son renforcement en raison même d'une certaine rigueur monastique mais les nouveaux maîtres conservent une vision très seigneuriale, l'église et son cimetière représentent encore des pouvoirs et des revenus âprement disputés à l'occasion. Si les moines renvoient le scandaleux Albéric de Fougères, ils conservent souvent le clergé en place, les deux fils de Main, guéris par l'abbé, donnent leurs deux églises mais demeurent en place ${ }^{87}$. Certains desservants qui se donnent avec leur église, se font moines tout en conservant sans doute des liens avec elle. À Erbrée, en 1104, deux religieux de Vitré sont agressés en pleine messe par une femme qui conteste la donation du sanctuaire. L'un des deux a toute chance d'être le donateur venu célébrer dans son église ${ }^{88}$. La question de la réforme du clergé n'est donc sans doute pas un objectif primordial - les chartes ne le montrent guère - mais sous l'impulsion de la gestion monastique une certaine évolution se produit à terme qui modifie sensiblement les données de la réalité et de la vie paroissiale.

Cependant, l'accentuation du caractère grégorien produit des effets inattendus. F. Mazel a bien étudié le lourd conflit qui oppose Marmoutier au seigneur de Fougères à propos du prieuré de la Trinité ${ }^{89}$. L'abbaye a visiblement cherché à contrôler l'ensemble de la vie religieuse de l'agglomération castrale au grand dam des chanoines de la collégiale seigneuriale. Le conflit aboutit à une rupture avec un seigneur pourtant soucieux de son salut et fondateur de l'abbaye de Savigny. Cet intérêt pour un nouvel ordre peut correspondre à une spiritualité plus séduisante mais on tient là un bon exemple de la rupture d'amicitia entre aristocratie et moines grégoriens. De tels conflits se rencontrent ailleurs mais surtout, le système des prieurés ne correspond plus vraiment à l'esprit du temps et la grande phase des fondations ne tarde pas à s'achever. Après 1130, une époque est close. Un autre monde se dessine, en ce sens, il y a bien crise du modèle des prieurés mais cela ne signifie en rien leur disparition ${ }^{90}$.

86. Colleter, Rozenn, Le Boulanger, Françoise, Рichot, Daniel, Église, cimetière et paroissiens. Bréal-sous-Vitré (Ille-et-Vilaine), étude historique, archéologique et anthropologique (VII'-XVIII siècle), Paris, 2012, chap. 3.

87. Dom Morice, Preuves, col. 491.

88. Arch. dép. d'Ille-et-Vilaine, 1F 544/5.

89. MAZEL, Florian, "Pouvoir local... ", op. cit.

90. LEGRos, Sébastien, Moines et seigneurs..., op. cit., chap. 11. 
En effet, au prix de quelques adaptations, les prieurés font mieux que survivre et leur place dans la société conserve une énorme importance en dehors aussi du domaine strictement religieux. L'exemple des bourgs en constitue sans doute le meilleur exemple. Les moines ont effectivement organisé nombre de ces lotissements, le plus souvent à l'ombre des châteaux, on n'en compte pas moins de dix huit dans ce cas ${ }^{91}$. Les plans cadastraux conservent la trace de ces plateae régulièrement disposées de part et d'autre d'une rue centrale comme à Vitré, Lamballe ou Jugon. Mais, si certains ne se développent que tardivement, d'autres ne tardent pas à montrer leur vigueur comme Sainte-Croix de Vitré qui s'élargit au-delà des limites initiales ${ }^{92}$. Les conséquences sont considérables. Ces bourgs, souvent couplés à un second bourg seigneurial, voire à plusieurs autres, contribuent au développement de véritables agglomérations castrales qui se concrétisent au cours du $\mathrm{XIII}^{\mathrm{e}}$ siècle et donnent naissance à un réseau urbain d'agglomérations secondaires, essentiel pour le monde des châtellenies et pour notre réseau urbain actuel. Rien qu'à énumérer la liste des maisons de Marmoutier, on constate son rôle considérable sur la carte de la Bretagne.

Les prieurés de Marmoutier offrent une riche approche des réalités bretonnes de la période féodale. L'ampleur des implantations met en lumière la place primordiale de l'abbaye dans le phénomène de reconstruction mais surtout d'organisation de la vie religieuse, en particulier dans l'établissement de rapports privilégiés avec l'aristocratie.

Le modèle monastique, séduit d'abord les princes dans la tradition carolingienne puis les barons principalement qui ont une vision plus spécifique liée à l'établissement de leur pouvoir. En ce sens, Marmoutier se présente bien comme l'abbaye par excellence des barons, politique voulue sans doute mais réussite aussi du projet monastique auprès des élites du pouvoir.

La force de ces implantations a des conséquences considérables sur l'organisation de la société, tant sur le plan religieux, que politique ou tout simplement économique et du peuplement. La force de la communauté monastique réside, bien sûr, dans l'abbaye mais on ne saurait sous-estimer l'importance que lui offre son réseau de prieurés. Au-delà de l’incontestable

91. HÉNOT, Aurélien, op. cit., p. 81 ; CHÉDEVILLE, André et PICHOT, Daniel (dir.), Des Villes à l'ombre des châteaux. Naissance et essor des agglomérations castrales en France au Moyen Âge, Rennes, 2010, p. 236-237.

92. Pichot, Daniel, " Naissance d'une ville ", op. cit., p. 18, 24. On sait maintenant que certains bourgs n'ont guère grandi et que d'autres se sont développés bien plus tard, ZADORA-RIo, Élisabeth et GAUTHIEZ, Bernard, "Les fondations de bourgs de l'abbaye de Marmoutier en Anjou-Touraine : ressorts de juridiction ou espace urbanisés? ", GAUTHIEZ, Bernard, ZADORA-RIo, Elisabeth, GaLINIE, Henri (dir.), Village et ville au Moyen Âge : les dynamiques morphologiques, Tours, 2003, p. 299-348. 
puissance du temporel qu'ils constituent, ils démultiplient la présence de l'idéal monastique à travers l'espace du duché.

\section{Annexe - Les prieurés de Marmoutier en Bretagne}

La liste des prieurés est établie par diocèse et chacun est suivi du code du département actuel où il se situe. Les dates proposées tiennent compte des travaux récents mais pour nombre de prieurés, les dates traditionnellement retenues seraient à vérifier ou revoir. Ce travail d'inventaire ne constitue qu'une première approche susceptible de révision.

\section{Diocèse de Rennes}

Fougères (La Trinité), Fougères, 1074-1076, 35.

Gahard (Saint-Exupère), 1015-1019, 35.

Louvigné-du-Désert? 1040-1047, 35.

Marcillé-Robert (Saint-Jacques), 1015-1032, 35.

Martigné-Ferchaud (Saint-Pierre), 1049-1064, 35.

Vitré (Sainte-Croix), 1063-1076, 35.

Saint-Ouen-la-Rouërie?, 1064-1066, 35.

Saint-Sauveur-des-Landes, 1040-1047, 35.

Sougéal (Saint-Jean-Baptiste), 1040-1047, 35.

\section{Diocèse de Nantes}

Chateaubriant (Saint-Jean de Béré), 1041, 44.

Champtoceaux (Saint-Jean-Baptiste), 1040-44, 49.

Donges (Notre-Dame), 1050-1052, 44.

Liré (Saint-Martin), vers 1059, 49.

Le Pellerin (Notre-Dame), vers 1050, 44.

Machecoul (Saint-Martin), avant 1115, 44.

Nantes (Sainte-Croix), 1106, 44.

Nort-sur-Erdre (Saint-Christophe), 1075, 44.

Pontchâteau (Saint-Martin), 1096, 44.

Varades (Saint-Pierre), vers 1050, 44.

\section{Diocèse de Saint-Malo}

Bécherel (Saint-Jacques), avant 1129, 35.

Combourg (Sainte-Trinité), 1047-1064, 35.

Dinan (Saint-Malo), avant 1108, 22.

Iffendic (Saint-Pierre), 1121, 35.

Josselin (Saint-Martin), 1108, 56.

Saint-Malo (de L'Isle), 1104-1108, 35. 


\section{Diocèse de Dol}

Léhon (Saint-Magloire), 1181, 22.

\section{Diocèse de Saint-Brieuc}

Jugon (Notre-Dame), 1104-1124, 22.

Lamballe (Saint-Martin), 1083, 22.

\section{Diocèse de Saint-Pol-de-Léon}

Morlaix (Saint-Martin), 1128, 29.

\section{Diocèse de Vannes}

Malestroit (La Madeleine), avant 1129, 56.

Rohan (Saint-Martin), 1128, 56.

\section{Diocèse de Quimper}

Île Tristan, 1118, 29.

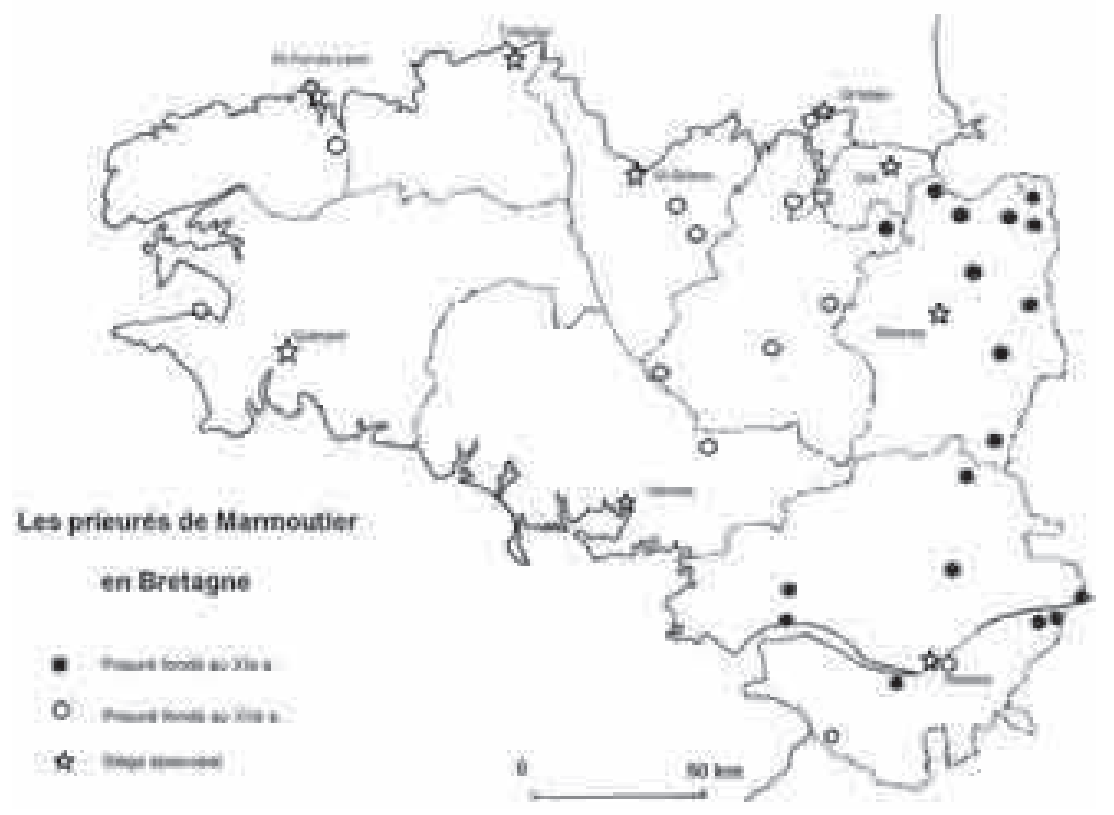

